\title{
The effect of two different types of forces on possible root resorption in relation to dentin phosphoprotein levels: a single-blind, split-mouth, randomized controlled trial
}

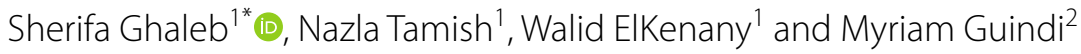

\begin{abstract}
Background: The purpose of this 2-arm-parallel split-mouth trial was to evaluate and compare the extent of possible root resorption using dentin phosphoprotein levels in gingival crevicular fluid between controlled continuous and intermittent orthodontic force groups.

Materials and methods: A sample of 16 maxillary first premolars from 8 patients requiring bilateral extractions of the upper first premolars as part of their orthodontic treatment were recruited. A buccally directed continuous force of $150 \mathrm{~g}$, reactivated after 28 days, was applied to the upper first premolar on one side for 8 weeks. On the contralateral first premolar, a buccally directed intermittent force (21 days on, 7 days off) of the same magnitude was applied for the same period. Gingival crevicular fluid samples were collected at the beginning of the study, 1st, 3rd, 4th and 5th week, and at the end of the study to quantify and compare dentin phosphoprotein levels in both groups.

Results: Dentin phosphoprotein levels showed a higher concentration in the continuous force group than the intermittent force group in week 4 and 8 of sample collection; where the differences were statistically significant $(95 \% \mathrm{Cl}$ $0.007-0.14 ; P<.04)$ and $(95 \% \mathrm{Cl} 0.02-0.17 ; P<.04)$ respectively. No harm was observed.

Conclusions: Dentin phosphoprotein was found to be a useful early biomarker to detect and monitor root resorption, showing that the application of an intermittent orthodontic force caused less root resorption than a continuous force.

Trial registration: NCT04825665 ClinicalTrials.gov. Registered 1 April 2021—Retrospectively registered, https://clinicaltr ials.gov/ct2/show/NCT04825665.
\end{abstract}

Keywords: Root resorption, Dentine phosphoprotein, Gingival crevicular fluid, Continuous force, Intermittent force

\section{Background}

One of the unfavorable sequelae of orthodontic treatment is root resorption [1]. Root resorption can be defined as a pathological or physiological process that leads to loss of cementum and dentine [2]. Commonly,

\footnotetext{
*Correspondence: sherifaadel.dent@alexu.edu.eg

${ }^{1}$ Department of Orthodontics, Faculty of Dentistry, Alexandria University,

Champolion street, Azarita, Egypt

Full list of author information is available at the end of the article
}

in orthodontics, it is referred to as an induced sterile inflammatory response which is a form of pathological external root resorption [1,3]. When orthodontic forces are transferred to the teeth, hyalinized areas in the periodontal spaces are formed. Thereafter, cells and blood vessels from the adjacent healthy periodontium lead to removal of the hyalinized tissues, this eventually results in loss of both cementoid and mature collagen, adjacent to the cementum. Subsequently, changes of the normal barriers to root resorption occur [2, 4]. However, root 
resorption resulting from orthodontic treatment is usually mild and clinically insignificant; although in some patients it can occur in large amounts [5].

Factors that have been linked to root resorption are genetic characteristics, biological factors, age of the patient and orthodontic treatment techniques. Orthodontic treatment techniques include the magnitude, duration and type of orthodontic force application [6-11]. In relevance to the manner of orthodontic force application, current data suggest that pausing orthodontic forces during treatment may reduce the amount of root resorption. This is likely due to cementum repair during the inactive periods $[8,9]$. Additionally, this is specifically important in individuals who are biologically and genetically prone to root resorption.

Early detection of teeth at risk of severe resorption is crucial [12]. Currently, using radiographs is common; however, they are technique sensitive, cannot indicate if the process of root resorption is still active and additional radiation exposure to the patient is a risk. Therefore, a safer, more reliable alternative method to clinically diagnose early stages of root resorption is much needed and may include detecting specific biomarkers in gingival crevicular fluid [13].

Gingival Crevicular Fluid (GCF) is the inflammatory transudate that flows out via the gingival crevice, known to contain an array of biochemical and cellular factors that reflect the state of the underlying periodontium [14]. Among the dentin breakdown products found in the GCF, that reflect loss of root structure is dentin phosphoprotein. Dentin phosphoprotein is a predominant non-collagenous protein that is cleaved from dentin sialophosphoprotein. Moreover, it accounts for approximately $50 \%$ of all the dentin non-collagenous proteins $[15,16]$.

\section{Specific objectives or hypotheses}

In this study the aim was to compare the extent of root resorption between continuous and intermittent orthodontic force groups, using the levels of dentin phosphoprotein in gingival crevicular fluid.

\section{Methods}

\section{Trial design}

This was a single-blind randomized controlled trial. It had a 2-arm split-mouth design in which the maxillary right first premolar of each patient was randomly allocated to either the continuous or intermittent group using randomization software (www.randomization.com) with a 1:1 allocation ratio, while the maxillary left first premolar was assigned to the other group. A split-mouth design was employed to control any potential patientrelated confounders such as root lengths, shapes, and age of patient. There were no alterations after commencement of the trial.

\section{Participants, eligibility criteria and settings}

Ethics approval was obtained from the scientific research ethics committee of Faculty of Dentistry, Alexandria University and informed oral and written consents were obtained from the patients' guardians.

Sixteen maxillary first premolars from eight participants ( 3 boys and 5 girls) aged between 13 and 18 years (mean age 15.8 years) who were recruited from the Outpatient Clinic at the Department of Orthodontics, Faculty of Dentistry, Alexandria University. These participants required bilateral maxillary first premolar extractions as part of their orthodontic treatment. Patient recruitment commenced in March 2020 and ended in December 2020. No previous reported or observed trauma, dental treatment or orthodontic treatment were considered to be the selection criteria for the teeth to be extracted. Subjects were excluded if they had past or present signs or symptoms of periodontal disease or bruxism, medical history that would affect the dentition and if apexification was not completed.

\section{Interventions}

In each subject, a buccally directed continuous tipping force of $150 \mathrm{~g}$ was applied to the maxillary first premolar (MFP) on one side for 8 weeks. On the contralateral side, a buccally directed intermittent force (21 days on, 7 days off) of the same magnitude was also applied for 8 weeks. The type of force application was assigned randomly to eliminate any allocation bias. A transpalatal arch was banded on the upper first molars to enhance the molar anchorage unit and ensure that the force applied to each experimental premolar was consistent. A 0.022in slot premolar bracket (Mini 2000, Ormco, California, United States) was bonded on the buccal surfaces of each MFP. The forces were induced using $0.017^{\prime \prime} \times 0.025^{\prime \prime}$ Titanium molybdenum alloy (TMA) cantilever spring (Beta III Titanium, 3 M Unitek, Monrovia, Calif) and the force magnitude was measured with a strain gauge (Morelli Ortodontia, Sorocaba, Brazil) (Fig. 1). On the continuous force side, the TMA springs were kept in place, checked and reactivated to the original force level on the 28th day of the experiment. While on the intermittent force side, a passive $0.017^{\prime \prime} \times 0.025^{\prime \prime}$ TMA wire (Beta III Titanium, 3 M Unitek, Monrovia, Calif) was placed during the rest periods to maintain the position of the upper first premolar. At the end of the 7 day rest period, the springs were placed again and the force level was calibrated to the original amount (Fig. 2).

Samples were collected from both experimental sites at the beginning of the study, 1st week, 3rd week, 4th 

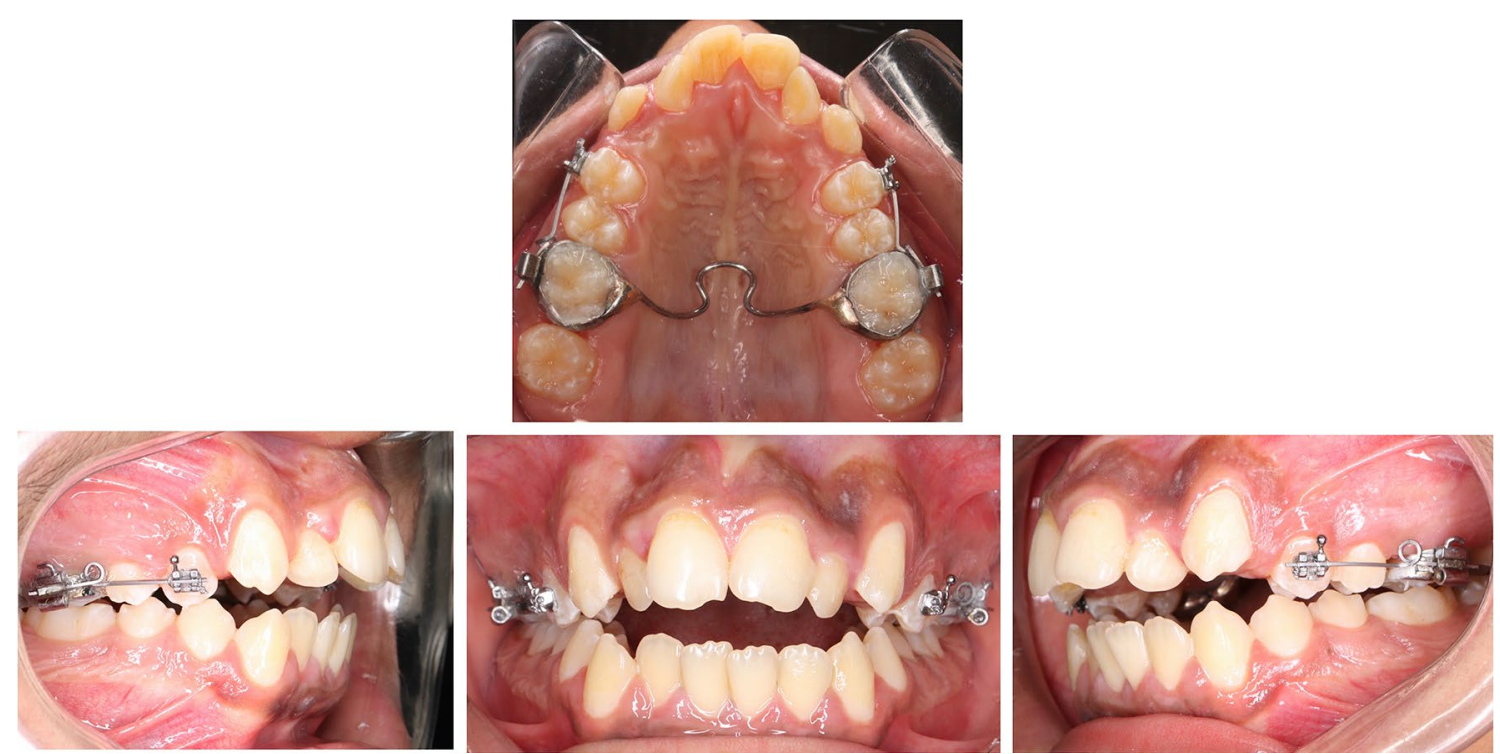

Fig. 1 Experimental appliance

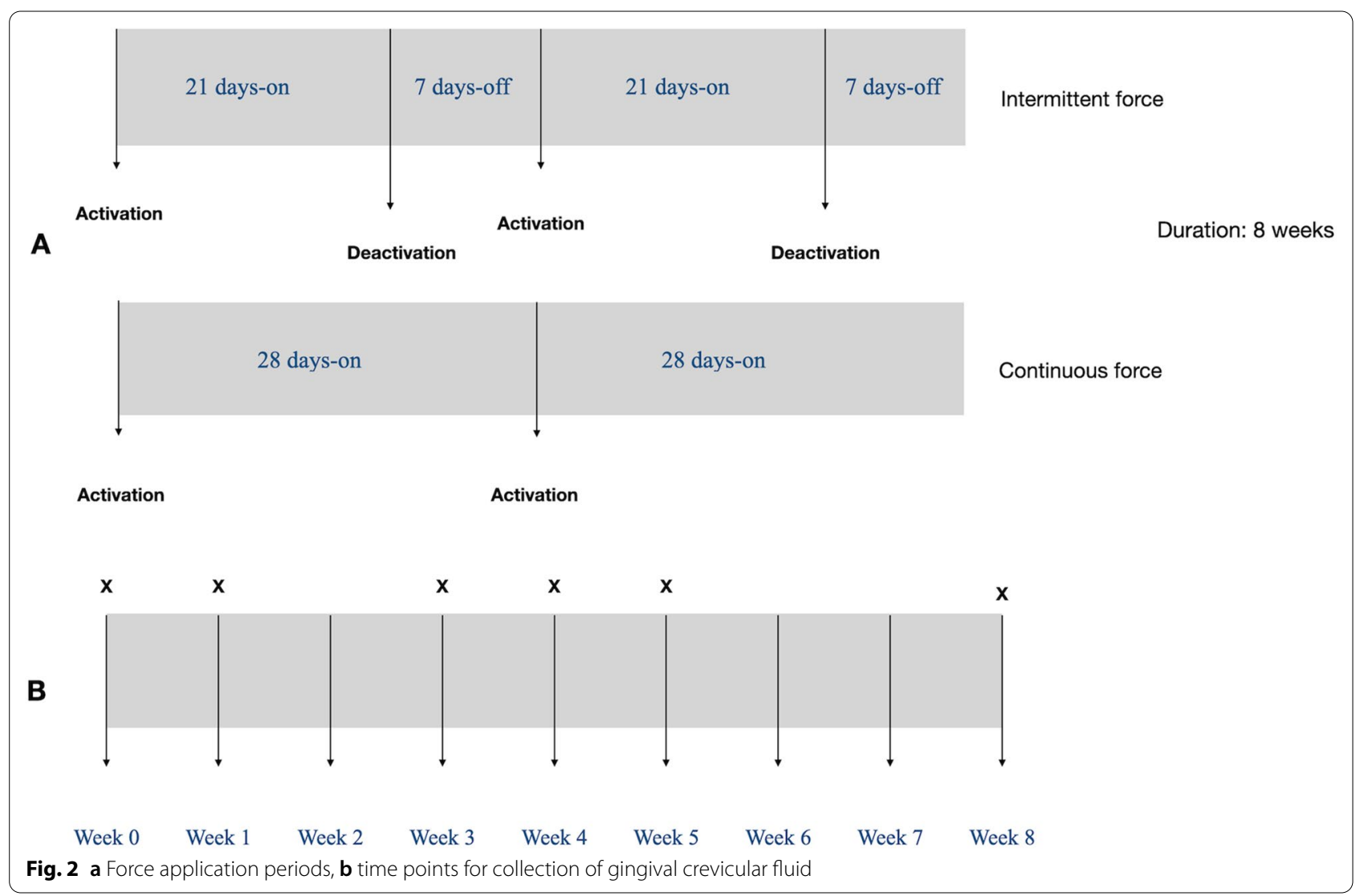

week, 5th week and at the end of the study period which is the 8th week (Fig. 2). Before the sample collection, each experimental tooth was isolated using a self-retaining retractor, suction, and cotton rolls, and gently dried with air for $5 \mathrm{~s}$. Standardized filter paper strips (Whatman International Ltd, Maidstone, UK), cut into standardized 
sizes $2 \times 10 \mathrm{~mm}$ were used for the collection of the gingival crevicular fluid samples. Each strip was inserted into the gingival crevice until mild resistance was felt and left insitu for $60 \mathrm{~s}$. After removal, new strips were placed at 1 min interval to obtain four strips at each site. Each set of 4 filter paper strips collected from the same experimental site were placed in an eppendorf tube containing $100 \mathrm{ul}$ of phosphate-buffered saline. It was then centrifuged at $3000 \times g$ for $10 \mathrm{~min}$ and stored at $-20^{\circ} \mathrm{C}$ for later analysis. Samples contaminated with saliva or blood were discarded and new samples were collected.

The samples were then assayed for dentin phosphoprotein using a dentin phosphoprotein (DPP) assay kit (Cloud clone, USA). Samples were analyzed using the enzyme linked immunosorbent assay (ELISA) method. The concentration of DPP in the samples was determined by comparing the optical density (O.D) of the samples to the standard curve. According to standards' concentrations and the corresponding OD values, the linear regression equation of the standard curve was calculated. Final results were reported as dentin phosphoprotein level ng/ ml per 60-s sample [17].

At the end of the experimental period, the experimental teeth were extracted with instructions to avoid forceps contact on the cervical cementum. They were immediately stored in marked tubes containing deionized water. The periodontal ligament was removed by using an ultrasonic bath for $10 \mathrm{~min}$, followed by disinfection in $70 \%$ alcohol for $30 \mathrm{~min}$, and then stored again in the deionized water. Teeth were bench dried before imaging [9].

The extracted teeth were scanned by a high resolution cone beam computed tomography system (Vatech Green CT, Korea) under a voxel size of 80 microns, a voltage of 95 kilovolts and current of 7 milliampere. After acquisition, the software ImageJ (Image), National Institute of
Health, USA) was used to measure the area of the root resorption craters. As for the volume estimation, Ondeman3dApp (Cybermed, Korea) was used for 3D STL file exporting, Exocad (Darmstadt, Germany) was used in reconstructing the resorption crater with a brush tool [18] and then lastly Materialise Magics 18.03 (Materialise, Belgium) was used to calculate the estimated volume of each resorption crater. This was done by measuring the volume of the tooth with the reconstructed crater and the original non constructed tooth, then subtracting them from each other to get the estimated volume of each resorption crater. Each resorption crater's position on the tooth was recorded (Fig. 3).

Maxillary dental plaster models were obtained before and after the experimental period and were scanned with an inEos X5 scanner (Dentsply, Sirona, USA). A reference line passing through the median palatine raphe was then constructed. The distances of the buccal cusps of the first premolars to the reference line were measured to calculate the buccal movement of each premolar and then comparison between both force groups was done (Fig. 4).

\section{Outcomes (primary and secondary) and any changes after trial commencement}

The main outcome of this study was to evaluate and compare the extent of root resorption between the continuous and intermittent orthodontic force groups using the levels of dentin phosphoprotein in gingival crevicular fluid. The secondary outcome was the three-dimensional comparison of the root resorption craters' volume and area in the extracted maxillary first premolar roots between both groups. The tertiary outcome was the comparison of amount of tooth movement during buccal tipping between both groups. There were no outcome changes after trial commencement.
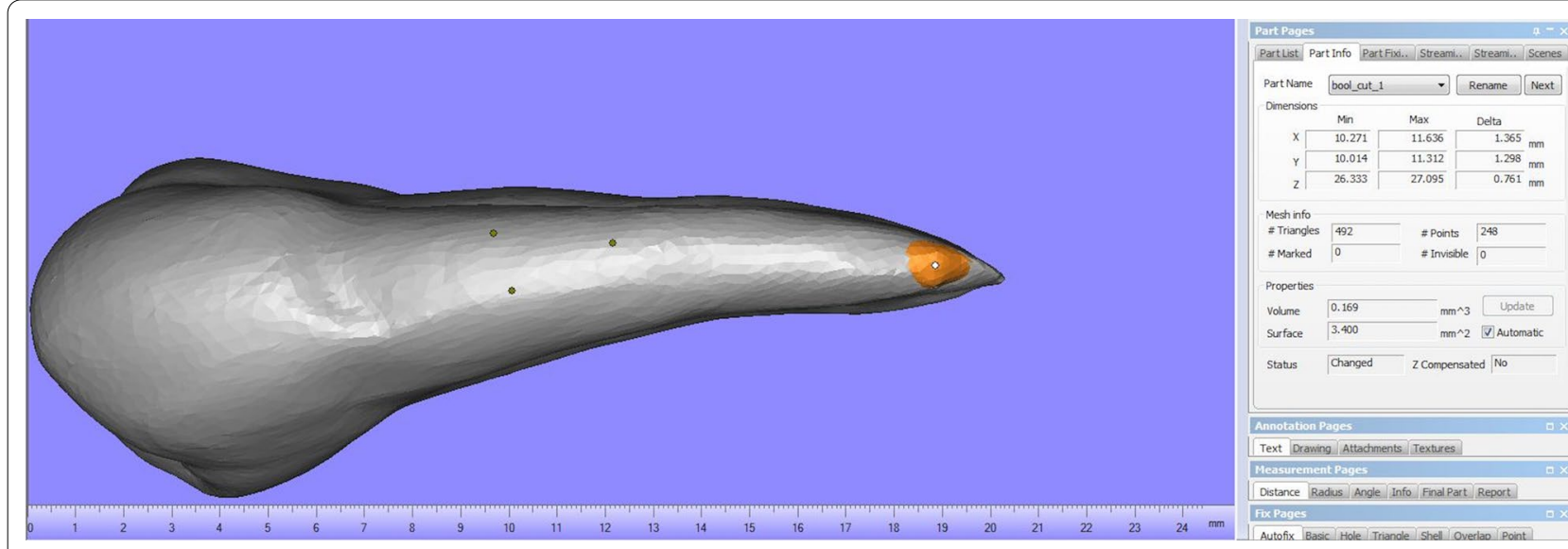

Fig. 3 Resorption crater 


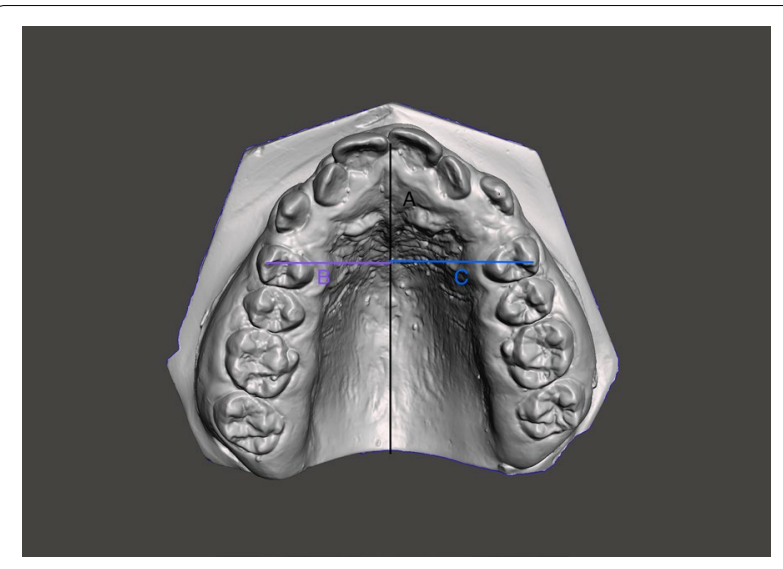

Fig. 4 Measurement of buccal movement of premolars. a Line demonstrating median palatine raphe; distances of $(\mathbf{b})$, the right premolar and $\mathbf{c}$ the left premolar to the (a) line

\section{Sample size calculation}

Sample size was estimated based on the following assumptions: alpha error $=5 \%$ and study power $=80 \%$. According to Ballard et al. [9] the mean cube root resorption volume after orthodontic movement $=0.815$, standard deviation (SD) calculated from the standard error $=0.099$ when intermittent orthodontic force was used, and mean $=0.985, \mathrm{SD}=0.099$ when continuous force was used. Dentin phosphoprotein in GCF is a biomarker for monitoring root resorption $[13,15]$. Based on comparison of means, sample size was calculated to be 7 per group and this was increased to 8 to make up for cases lost to follow-up. Therefore total sample size $=16$ [19].

\section{Randomization and blinding}

The right first maxillary premolar from each patient was randomly allocated to either the continuous or intermittent group using randomization software (www. randomization.com) with a 1:1 allocation ratio. The left first maxillary premolar was then assigned to the alternate group. Allocation concealment was achieved with sequentially numbered, opaque, sealed envelopes containing the treatment allocation cards, which were prepared before the trial. The primary investigator was responsible for opening the next envelope in sequence and implementing the randomization process. Blinding of either patient or operator was not possible; however, blinding during outcome assessment was. This was because it was difficult to distinguish between the treatment groups during outcome assessment from the eppendorf tubes collected, the teeth scanned or the models analyzed.

\section{Statistical analysis}

Normality was checked for all variables using descriptive statistics, normality tests and plots (boxplot and histogram). All variables showed non-normal distribution, so non-parametric analysis was adopted. Comparison of biomarker level, amount of tooth movements and crater volume between the two study groups was done using Wilcoxon signed rank test. Mean differences and 95\% confidence intervals (CI) were calculated. Comparison of different timepoints for the biomarker level, and different regions of craters within each group were done using Friedman test, followed by multiple pairwise comparisons using Bonferroni adjusted significance level. Spearman correlation was performed to assess the relation between DPP levels at 8 weeks and total amount of root resorption in the two study groups. Significance was set at $P$ value $<0.05$ and data were analyzed using IBM SPSS for Windows (Version 23.0).

\section{Results}

\section{Participant flow}

Patient flow through the study is illustrated in the CONSORT diagram (Fig. 5). Sixteen maxillary first premolars from eight orthodontic patients, which included 3 males (33\%), 5 females (66\%) with a mean age of $(15.88 \pm 2.03)$ years were randomized in a 1:1 ratio to either continuous or intermittent force group in the same patient. No patients were lost to follow-up. At baseline, at week 0 , dentin phosphoprotein levels were similar on both sides $(0.021 \pm 0.009$ and $0.020 \pm 0.008 \mathrm{ng} / \mathrm{ml}$ per 60 -s sample) .

\section{Numbers analyzed for each outcome, estimation and precision, subgroup analyses}

The sixteen maxillary first premolars randomly assigned to each group were all included in the analyses done. Dentin phosphoprotein levels showed a mean difference change from week 0 to week 8 of $(0.370 \pm 0.01)$ and $(0.273 \pm 0.09)$ in the continuous and intermittent groups respectively. However, it was higher in the continuous group with a statistical significant difference $(95 \% \mathrm{CI}$ $0.02-0.18 ; P<0.05)$. DPP levels showed a statistically significant higher concentration in the continuous group than the intermittent group in weeks 4 and $8(P<0.05)$ (Table 1) (Fig. 6).

Intragroup comparisons for both groups revealed that DPP levels at all time points compared to week 0 were higher, with a statistical significant difference in week 1,3 , 5 for both groups. While week $8(0.391 \pm 0.002)$ was statistically significantly higher than week $0(0.021 \pm 0.009)$ $(P<0.001)$ for the continuous group only. Also for the 


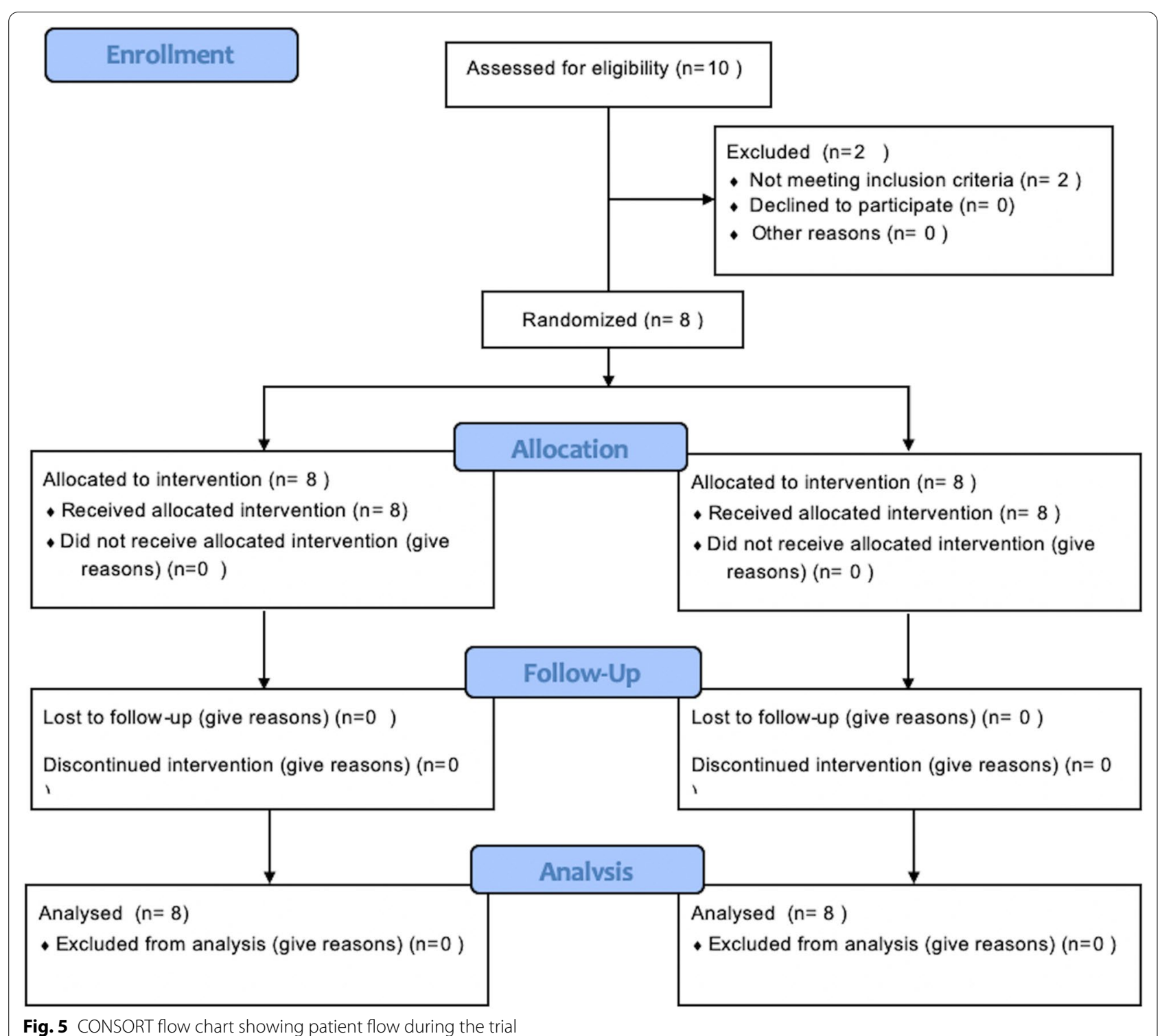

Fig. 5 CONSORT flow chart showing patient flow during the trial

continuous group only, week 8 was statistically significantly higher than week $4(P<0.01)$ (Table 2$)$.

The mean resorption crater area for the continuous group was higher than the intermittent force group with a statistical significant difference $(P<0.05)$. The mean resorption crater volume (MRCV) for the continuous force group $\left(0.13 \pm 0.06 \mathrm{~mm}^{3}\right)$ was higher than the intermittent force group $\left(0.05 \pm 0.03 \mathrm{~mm}^{3}\right)$. This difference was statistically significant as well $(95 \% \mathrm{CI}, 0.06-0.10$; $P<0.001$ ) (Table 3) (Fig. 7).

Results for the different tooth surfaces showed that the MRCVs on the buccal and mesial surfaces especially were statistically significantly greater in the continuous force group $(P<0.01)$ and $(P<0.05)$ respectively. While the results for different root levels showed that at the cervical and apical thirds, the continuous force group displayed greater MRCVs with a statistically significant difference, $(P<0.01)$ and $(P<0.05)$ respectively (Table 3$)$ (Fig. 7). The buccal cervical region was the only region which displayed statistically significantly higher MRCVs in the continuous force group compared to the intermittent force group $(P<0.01)$ (Table 4$)$.

There was a moderate direct significant correlation between DPP levels at 8 weeks and total root resorption volume in the continuous force group $(\mathrm{Rho}=0.44$, $P=0.01$ ), and a weak direct correlation between DPP levels and total root resorption in the intermittent force group (Rho $=0.36, P=0.02)$ (Table 5 ). 
Table 1 Dentin phosphoprotein biomarker levels in the two study groups at the different time points

\begin{tabular}{|c|c|c|c|c|c|}
\hline & Continuous force & Intermittent force & Mean difference (SD) & $95 \% \mathrm{Cl}$ & $\begin{array}{l}\text { WSR } \\
P \text { value }\end{array}$ \\
\hline \multicolumn{6}{|l|}{ Week 0} \\
\hline Mean $\pm S D$ & $0.021 \pm 0.009$ & $0.020 \pm 0.008$ & $0.001(0.01)$ & $-0.01,0.01$ & 0.78 \\
\hline Median (IQR) & $0.021(0.012,0.030)$ & $0.021(0.011,0.028)$ & & & \\
\hline \multicolumn{6}{|l|}{ Week 1} \\
\hline Mean \pm SD & $0.388 \pm 0.007$ & $0.383 \pm 0.005$ & $0.005(0.006)$ & $-0.0007,0.01$ & 0.13 \\
\hline Median (IQR) & $0.386(0.383,0.387)$ & $0.383(0.382,0.385)$ & & & \\
\hline \multicolumn{6}{|l|}{ Week 3} \\
\hline Mean \pm SD & $0.384 \pm 0.003$ & $0.388 \pm 0.005$ & $-0.004(0.006)$ & $-0.009,0.0009$ & 0.08 \\
\hline Median (IQR) & $0.383(0.383,0.385)$ & $0.389(0.387,0.392)$ & & & \\
\hline \multicolumn{6}{|l|}{ Week 4} \\
\hline Mean \pm SD & $0.375 \pm 0.002$ & $0.299 \pm 0.08$ & $0.07(0.08)$ & $0.007,0.14$ & $0.04^{*}$ \\
\hline Median (IQR) & $0.375(0.374,0.375)$ & $0.294(0.267,0.368)$ & & & \\
\hline \multicolumn{6}{|l|}{ Week 5} \\
\hline Mean \pm SD & $0.385 \pm 0.004$ & $0.340 \pm 0.07$ & $0.04(0.07)$ & $-0.01,0.10$ & 0.21 \\
\hline Median (IQR) & $0.385(0.382,0.388)$ & $0.367(0.263,0.394)$ & & & \\
\hline \multicolumn{6}{|l|}{ Week 8} \\
\hline Mean \pm SD & $0.391 \pm 0.002$ & $0.293 \pm 0.09$ & $0.10(0.09)$ & $0.02,0.17$ & $0.04^{*}$ \\
\hline Median (IQR) & $0.391(0.390,0.392)$ & $0.292(0.202,0.392)$ & & & \\
\hline \multicolumn{6}{|c|}{ Mean difference (week 8-0) } \\
\hline Mean \pm SD & $0.370 \pm 0.01$ & $0.273 \pm 0.09$ & $0.10(0.09)$ & $0.02,0.18$ & $0.04^{*}$ \\
\hline Median (IQR) & $0.372(0.360,0.380)$ & $0.271(0.180,0.373)$ & & & \\
\hline Friedman test & $<0.001^{*}$ & $<0.001^{*}$ & & & \\
\hline
\end{tabular}

WSR Wilcoxon signed rank test, $S D$ standard deviation, $\mathrm{Cl}$ confidence interval

*Statistically significant at $P$ value $<0.05$

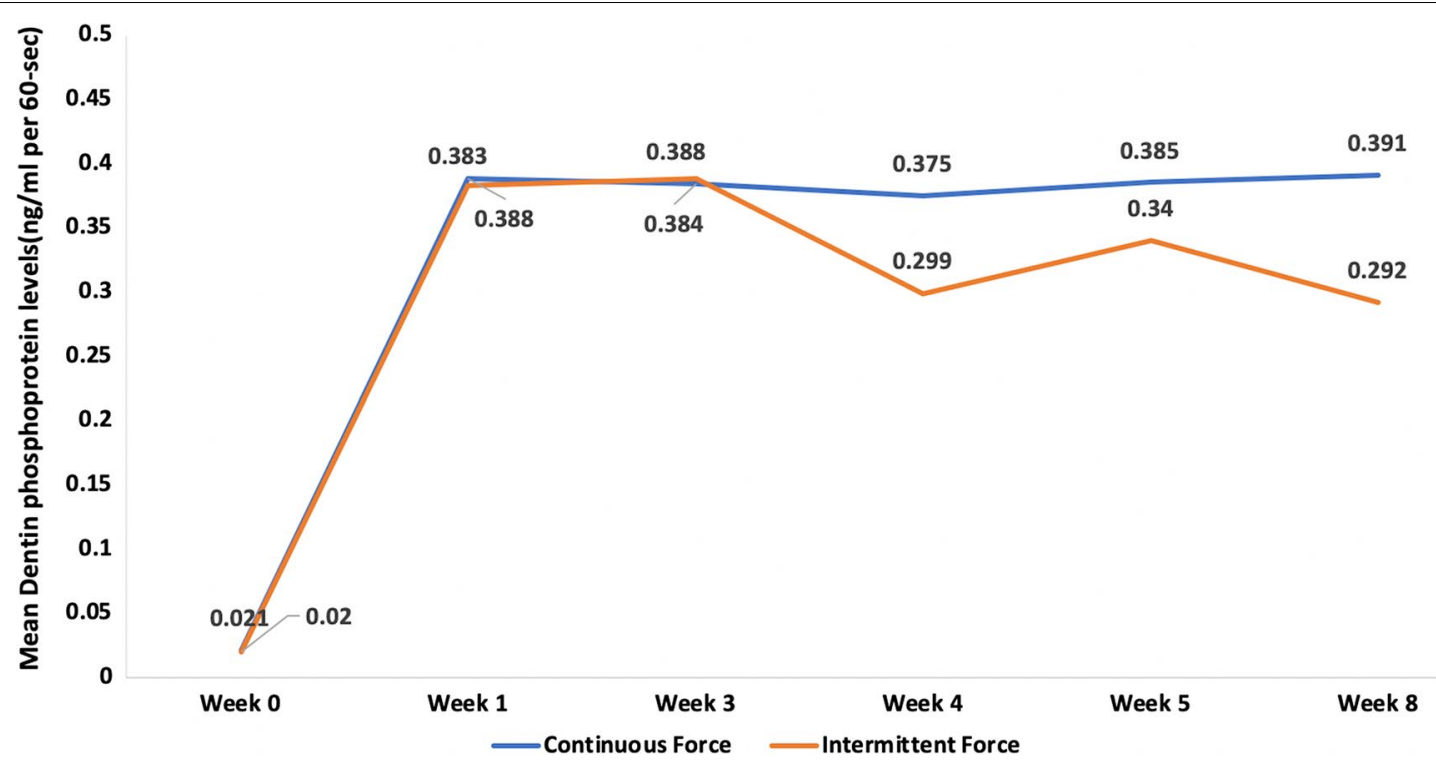

Fig. 6 Dentin phosphoprotein biomarker levels at different time points in the two study groups 
Table 2 Post-hoc pairwise comparisons of biomarker levels in each group using Bonferroni adjusted significance levels

\begin{tabular}{llll}
\hline Time point & Compared to & $\begin{array}{l}\text { Continuous force } \\
\text { P value }\end{array}$ & $\begin{array}{l}\text { Intermittent force } \\
\text { P value }\end{array}$ \\
\hline Week 0 & Week 1 & $0.006^{*}$ & $0.008^{*}$ \\
& Week 3 & $0.049^{*}$ & $<0.001^{*}$ \\
& Week 4 & 1.00 & 0.68 \\
& Week 5 & $0.008^{*}$ & $0.01^{*}$ \\
Week 1 & Week 8 & $<0.001^{*}$ & 0.14 \\
& Week 3 & 1.00 & 1.00 \\
& Week 4 & 0.20 & 1.00 \\
& Week 5 & 1.00 & 1.00 \\
Week 3 & Week 8 & 1.00 & 1.00 \\
& Week 4 & 0.92 & 0.17 \\
& Week 5 & 1.00 & 1.00 \\
Week 4 & Week 8 & 0.58 & 0.79 \\
& Week 5 & 0.24 & 1.00 \\
Week 5 & Week 8 & $0.001^{*}$ & 1.00 \\
\hline
\end{tabular}

*Statistically significant difference using Bonferroni adjusted significance level

The amount of buccal tooth movement was statistically significantly greater in the continuous force group compared with the intermittent force group with a mean difference of $1.13(P<0.05)$ (Table 6).

Harms

There were no reported harms or adverse effects.

\section{Discussion}

Commonly, radiographs are used to diagnose root resorption. However, it was found that $60-70 \%$ of mineralized tooth structure would have already been lost. Additionally, radiographs are unable to indicate if the process of root resorption is still active and exposes patients to unnecessary radiation exposure [13]. Alternatively, biomarkers have been suggested, in limited studies, to be a reliable method to clinically diagnose early stages of root resorption. Thus this study evaluated and compared the possible effects of continuous and intermittent orthodontic forces on root resorption by assessing dentin phosphoprotein levels.

Results of a systematic review by Tarallo et al. [20] compared different biomarkers in the GCF to detect root resorption in patients undergoing orthodontic treatment. They concluded that DPP was the most specific biomarker for early diagnosis of root resorption and suggested it to be used in future clinical trials. Also, it was found that cementum proteins are not highly indicative of the actual root structure loss because some areas of the cementum can be resorbed and repaired during orthodontic tooth movement. Therefore, previous studies suggested that the most indicative markers for the diagnosis of root resorption were dentin specific proteins only. Proteins like dentin sialoprotein have been suggested to be less indicative, since they were found to be present in both the root cement matrix and the dentin [20,21]. Consequently, dentin phosphoprotein was selected as the biomarker for this study.

Multiple findings in this study showed that DPP may be an excellent biomarker for detecting root resorption. Both groups, at week 1 and 3 when compared to

Table 3 Root resorption craters area and volume in the two study groups

\begin{tabular}{|c|c|c|c|c|c|}
\hline & $\begin{array}{l}\text { Continuous force } \\
\text { Mean } \pm \text { SD }\end{array}$ & Intermittent force & Mean difference (SD) & $95 \% \mathrm{Cl}$ & $\begin{array}{l}\text { WSR } \\
P \text { value }\end{array}$ \\
\hline Crater area & $1.92 \pm 0.82$ & $1.48 \pm 0.34$ & $0.43(1.01)$ & $0.06,0.08$ & $0.03^{*}$ \\
\hline Total crater volume & $0.13 \pm 0.06$ & $0.05 \pm 0.03$ & $0.08(0.06)$ & $0.06,0.10$ & $<0.001^{*}$ \\
\hline Total buccal volume & $0.14 \pm 0.07$ & $0.04 \pm 0.03$ & $0.10(0.07)$ & $0.05,0.14$ & $0.003^{*}$ \\
\hline Total lingual volume & $0.14 \pm 0.02$ & $0.07 \pm 0.04$ & $0.07(0.06)$ & $-0.02,0.16$ & 0.07 \\
\hline Total mesial volume & $0.15 \pm 0.05$ & $0.05 \pm 0.04$ & $0.11(0.06)$ & $0.05,0.18$ & $0.03^{*}$ \\
\hline Total distal volume & $0.06 \pm 0.001$ & $0.07 \pm 0.02$ & $-0.02(0.03)$ & $-0.26,0.22$ & 0.18 \\
\hline Total cervical volume & $0.16 \pm 0.06$ & $0.05 \pm 0.04$ & $0.11(0.07)$ & $0.06,0.15$ & $0.002^{*}$ \\
\hline Total middle volume & $0.08 \pm 0.02$ & $0.04 \pm 0.02$ & $0.04(0.03)$ & $-0.03,0.10$ & 0.11 \\
\hline Total apical volume & $0.14 \pm 0.02$ & $0.05 \pm 0.02$ & $0.09(0.03)$ & $0.06,0.12$ & $0.03^{*}$ \\
\hline \multicolumn{6}{|l|}{ Friedman test } \\
\hline Buccal-lingual-mesial-distal & 0.31 & 0.20 & & & \\
\hline Cervical-middle-apical & 0.10 & 0.69 & & & \\
\hline
\end{tabular}

WSR Wilcoxon signed rank test, SD standard deviation, $\mathrm{Cl}$ confidence interval

*Statistically significant at $P$ value $<0.05$ 


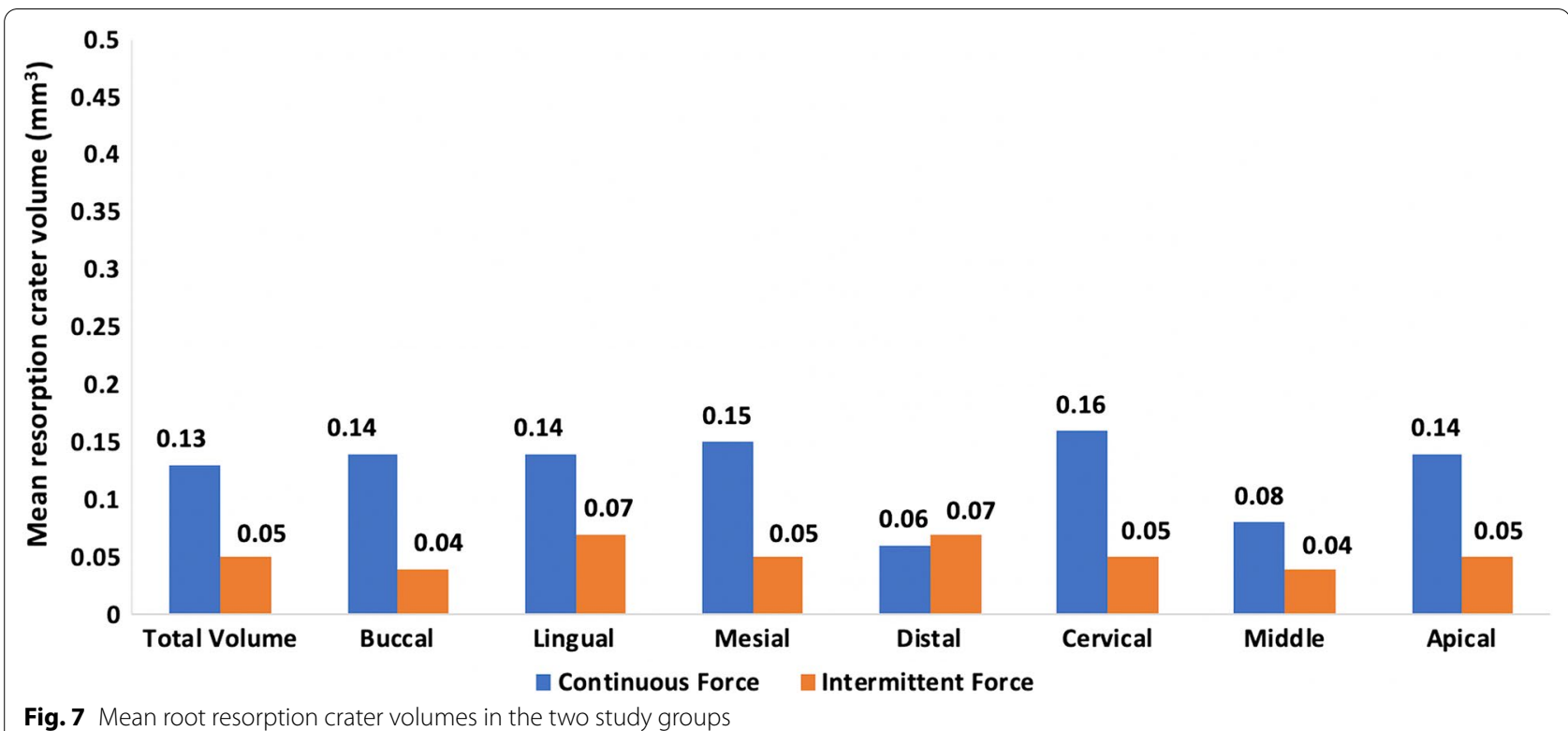

Table 4 Root resorption crater volume at different regions in the two study groups

\begin{tabular}{|c|c|c|c|c|c|}
\hline & $\begin{array}{l}\text { Continuous force } \\
\text { Mean } \pm \text { SD }\end{array}$ & Intermittent force & Mean difference (SD) & $95 \% \mathrm{Cl}$ & $\begin{array}{l}\text { WSR } \\
P \text { value }\end{array}$ \\
\hline \multicolumn{6}{|l|}{ Buccal } \\
\hline Cervical & $0.15 \pm 0.08$ & $0.05 \pm 0.03$ & $0.10(0.08)$ & $0.03,0.16$ & $0.008^{*}$ \\
\hline Middle & $0.06 \pm 0.008$ & $0.005 \pm 0.0007$ & $0.06(0.007)$ & $-0.003,0.12$ & 0.18 \\
\hline Apical & $0.06 \pm 0.002$ & $0.06 \pm 0.001$ & $0.0002(0.0007)$ & $-0.004,0.009$ & 0.18 \\
\hline P value & 0.16 & 0.22 & & & \\
\hline \multicolumn{6}{|l|}{ Lingual } \\
\hline Cervical & $0.16 \pm 0.02$ & $0.11 \pm 0.001$ & $0.05(0.02)$ & $-0.16,0.25$ & 0.18 \\
\hline Middle & $0.14 \pm 0.00$ & $0.004 \pm 0.00$ & $0.14(0.00)$ & $-0.001,0.001$ & 0.18 \\
\hline Apical & $0.14 \pm 0.02$ & $0.03 \pm 0.002$ & $0.12(0.03)$ & $-0.12,0.35$ & 0.18 \\
\hline$P$ value & 0.37 & 0.37 & & & \\
\hline \multicolumn{6}{|l|}{ Mesial } \\
\hline Cervical & $0.18 \pm 0.02$ & $0.06 \pm 0.06$ & $0.14(0.05)$ & $-0.06,0.21$ & 0.07 \\
\hline Middle & $0.09 \pm 0.0007$ & $0.05 \pm 0.04$ & $0.05(0.02)$ & $-0.12,0.22$ & 0.18 \\
\hline Apical & $0.06 \pm 0.001$ & $0.06 \pm 0.02$ & $0.0004(0.03)$ & $0.25,0.26$ & 0.66 \\
\hline$P$ value & 0.14 & 0.17 & & & \\
\hline \multicolumn{6}{|l|}{ Distal } \\
\hline Cervical & $0.12 \pm 0.01$ & $0.11 \pm 0.001$ & $0.01(0.02)$ & $-0.13,0.15$ & 0.18 \\
\hline Middle & $0.06 \pm 0.001$ & $0.05 \pm 0.001$ & $0.001(0.00)$ & $-0.01,0.01$ & 0.16 \\
\hline Apical & $0.07 \pm 0.001$ & $0.07 \pm 0.0007$ & $0.001(0.002)$ & $-0.02,0.02$ & 0.32 \\
\hline$P$ value & 0.14 & 0.14 & & & \\
\hline
\end{tabular}

WSR Wilcoxon signed rank test, $S D$ standard deviation, $C l$ confidence interval

*Statistically significant at $P$ value $<0.05$

week 0 had statistically significantly higher DPP levels. This could be explained by the start of root resorption once the $150 \mathrm{~g}$ force was applied. Moreover until week 3 , dentin phosphoprotein levels increased in both force groups with no statistical significant difference between them, since both had the force adjusted at the same level $(150 \mathrm{~g})$ at the start. Furthermore, in weeks 4 and 8 and the mean difference calculated between week 8 and week 
Table 5 Correlation between DPP level at 8 weeks and total root resorption volume in the two study groups

\begin{tabular}{lll}
\hline & $\begin{array}{l}\text { Correlation coefficient } \\
\text { (Rho) }\end{array}$ & $P$ value \\
\hline Continuous force & 0.44 & $0.01^{*}$ \\
Intermittent force & 0.36 & $0.02^{*}$ \\
\hline
\end{tabular}

*Statistically significant at $P$ value $<0.05$

0 , a statistically significant difference in the DPP level between the continuous and intermittent force group was found. This suggested that DPP could detect the difference in root resorption between the two different groups. This was in agreement with the study done by Mah et al. [15] which showed that there is a statistically significant difference between a control group, the roots of the second primary molars undergoing resorption and an orthodontic group. Balducci et al. [13] achieved similar results, where concentrations of DPP were highest in the group with severe root resorption followed by the mild resorption group and the control group.

By week 4, after the 7 day rest period for the intermittent group, the continuous force group exhibited a significantly higher level of DPP. This corroborated the fact that cementum has a repair potential when a pause of force occurs resulting in less progressive root resorption. Again, this was in agreement with several studies $[8,9,22]$. This also occurred at week 8 , for the second cycle; suggesting that the intermittent force manner may be beneficial for certain patients who are at high risk of root resorption. At week 5, DPP in the intermittent group started to increase again since the force level was reactivated after 28 days for both groups. Presence of DPP at Week 0 when teeth were not subjected to any orthodontic forces yet, was explained previously by Balducci et al. [13] and Mah et al. [15]. They found that the most reasonable explanation to this finding could be attributed to the sensitivity of the ELISA method.

This intermittent regimen of a 7 day rest period was chosen in accordance to a previous study by Ozkalayci et al. [8] since a 3-day inactive period applied by two previous studies meant that the patients had to attend their orthodontic adjustment appointments more frequently, which could be inconvenient to the patients and clinicians $[9,23]$. Moreover, from a biological standpoint, the longer rest period would allow more time for the recruitment of cementoblasts and initiation of the reparative process. Moreover, since generally clinicians see patients at 4- to 6-week intervals for their orthodontic adjustment, we opted for a 8 week (56 days) total study period in order to mimic a 4 week interval patient visit, repeating it over 2 cycles. This also accommodates for patients who might require more than 10 to 35 days for the resorption craters to appear as Ballard et al. [9] explained in their study with a 8 week study period as well.

In this study high resolution cone beam computed tomography was used which followed the suggestions of several previous studies that compared different voxel sizes of cone beam computed tomography (CBCT) in the diagnosis of external root resorption. High resolution CBCT with a smaller voxel size has been suggested to be used when one intends to investigate the early stages of external root resorption $[24,25]$. Mean root crater area and $\mathrm{MRCV}$ in the intermittent force group when compared to the continuous force group were significantly less, which is in agreement with the results of other studies $[8,23]$. This finding also suggests that the 1 -week inactive period permits the reversible defense mechanism to initiate the reparative process on the root surface [26].

The MRCVs on the buccal surfaces and the mesial surfaces, specifically, were significantly greater in the continuous force group. This conformed with the results and explanation of another study which stated that the force direction produced by the spring led to buccal tipping movement which may have induced a pressure zone at the buccal side [23]. Over and above, the premolar morphology and the rotational nature of the cantilever spring, may have been the cause of the pressure zone detected on the mesial surface [8]. The buccal tipping movement may also explain the significantly higher MRCV at the buccal cervical region in the continuous force group. While the intermittent force group could have experienced all the previous, however it had the time to repair, explaining the significant difference.

Ozkalayci et al. [8] found that MRCV in the intermittent force group was greater at the apical level, while our study found the opposite. This may be explained by

Table 6 Amount of tooth movement during buccal tipping in the two study groups

\begin{tabular}{llll}
\hline & Continuous force & Intermittent force & Mean difference (SD) \\
\hline Mean \pm SD & $2.13 \pm 1.55$ & $1.00 \pm 0.84$ & $1.13(1.19)$ \\
Median $(I Q R)$ & $2.00(0.50,3.75)$ & $0.75(0.50,1.75)$ & $0.13,2.12$ \\
\hline
\end{tabular}

WSR Wilcoxon signed rank test, SD standard deviation, $\mathrm{Cl}$ confidence interval

*Statistically significant at $P$ value $<0.05$ 
the passive wire placed during the 7-day inactive periods in our study in order to retain the buccally tipped maxillary first premolar in place, which was suggested but not placed by the other study. Hence, when applying intermittent force during orthodontic treatment, it is important to have a passive archwire in place during the inactive phases to avoid relapse and pressure zones at the apical area. Continuous force application resulted in greater tooth movement, as shown by the buccal cusp movement, which correlated with previous studies $[8$, 23].

\section{Limitations}

The Systematic review by Tarallo et al. [20] suggested to design new clinical trials focusing on the ability of GCF to diagnose root resorption early and to prefer the DPP as a biomarker. Since the previous studies using DPP to detect root resorption were only two $[13,15]$. This study is also only the beginning. Further investigations with a larger sample size and using more sensitive DPP kits, if produced in the future, will be beneficial to overcome the shortcomings of the present investigation and highlight the clinical relevance as well. Lastly, a shortcoming of this study may be due to salivary contamination of the GCF collected; however, special care was taken to discard any samples contaminated with saliva or blood and new samples were taken immediately.

\section{Generalizability}

This study showed that dentin phosphoprotein in gingival crevicular fluid can help patients who are genetically and/or biologically prone to root resorption, since it can diagnose early root resorption and can monitor it throughout the orthodontic treatment. Also, for these patients in specific, the intermittent force regimen was found to be a convenient and effective way to move teeth with less damaging effects on the root surface.

\section{Conclusions}

Based on the findings of this randomized clinical trial, it was concluded that:

1. Dentin phosphoprotein was found to be a useful biomarker for detecting and monitoring root resorption. Patients who are prone and predisposed to orthodontically induced inflammatory root resorption may benefit from the biomarker's early, radiation free diagnostic ability.

2. Intermittent orthodontic force caused less root resorption than the continuous orthodontic force, in relevance to dentin phosphoprotein levels and mean volume estimation of root resorption craters.
3. The efficiency of tooth movement was compromised with the intermittent force group compared to the continuous force group.

\section{Abbreviations}

GCF: Gingival Crevicular Fluid; MFP: Maxillary first premolar; TMA: Titanium molybdenum alloy; DPP: Dentin phosphoprotein; ELISA: Enzyme linked immunosorbent assay; O.D: Optical Density; SD: Standard deviation; Cl: Confidence intervals; MRCV: Mean resorption crater volume; CBCT: Cone beam computed tomography.

\section{Acknowledgements}

The authors appreciate the beneficial assistance of the Department of Clinical Pathology at Alexandria University for conducting the laboratory analyses.

\section{Authors' contributions}

SG aided in the conceptualization, methodology, investigation, resources, writing the original draft of the research. NT helped in the methodology, validation, reviewing and editing, and supervision of the work. WK helped in conceptualization, methodology, validation, reviewing and editing and supervision. MG aided in the methodology, supervision of the laboratory results. All authors read and approved the final manuscript.

\section{Funding \\ Self-funded.}

\section{Availability of data and materials}

The datasets used and/or analyzed during the current study are available from the corresponding author on reasonable request.

\section{Declarations}

Ethics approval and consent to participate

Informed oral and written consents were obtained from the patients' guardians. The scientific research ethics committee of Faculty of dentistry, Alexandria university approved this scientific research. International number IORG0008839 and Ethics committee number 0125-03/2020.

\section{Consent for publication}

Written informed consent for publication of the patient's clinical intraoral images was obtained from the guardian of the patient. A copy of the consent form is available for review.

\section{Competing interests}

The authors declare that they have no competing interests.

\section{Author details}

${ }^{1}$ Department of Orthodontics, Faculty of Dentistry, Alexandria University, Champolion street, Azarita, Egypt. ${ }^{2}$ Department of Clinical Pathology, Faculty of Medicine, Alexandria University. Champolion Street, Azarita, Egypt.

Received: 17 August 2021 Accepted: 26 October 2021

Published online: 20 December 2021

\section{References}

1. Brezniak N, Wasserstein A. Root resorption after orthodontic treatment: Part 1. Literature review. Am J Orthod Dentofacial Orthop. 1993;103(1):62-6.

2. Ramanathan C, Hofman Z. Root resorption in relation to orthodontic tooth movement. Acta Med Hradec Kralove. 2006;49(2):91.

3. Dindaroğlu F, Doğan S. Root resorption in orthodontics. Turk J Orthod. 2016;29(4):103. 
4. Blake M, Woodside DG, Pharoah MJ. A radiographic comparison of apical root resorption after orthodontic treatment with the edgewise and Speed appliances. Am J Orthod Dentofacial Orthop. 1995;108(1):76-84.

5. Kaley J, Phillips C. Factors related to root resorption in edgewise practice. Angle Orthod. 1991;61(2):125-32.

6. Brezniak N, Wasserstein A. Orthodontically induced inflammatory root resorption. Part II: the clinical aspects. Angle Orthod. 2002;72(2):180-4.

7. Linge $B O$, Linge L. Apical root resorption in upper anterior teeth. Eur J Orthod. 1983;5(3):173-83.

8. Ozkalayci N, Karadeniz El, Elekdag-Turk S, Turk T, Cheng LL, Darendeliler MA. Effect of continuous versus intermittent orthodontic forces on root resorption: a microcomputed tomography study. Angle Orthod. 2018;88(6):733-9.

9. Ballard DJ, Jones AS, Petocz P, Darendeliler MA. Physical properties of root cementum: part 11. Continuous vs intermittent controlled orthodontic forces on root resorption. A microcomputed-tomography study. Am J Orthod Dentofacial Orthop. 2009;136(1):8-e1.

10. Chan E, Darendeliler MA. Physical properties of root cementum: Part 5. Volumetric analysis of root resorption craters after application of light and heavy orthodontic forces. Am J Orthod Dentofacial Orthop. 2005;127(2):186-95.

11. Weltman B, Vig KWL, Fields HW, Shanker S, Kaizar EE. Root resorption associated with orthodontic tooth movement: a systematic review. Am J Orthod Dentofacial Orthop. 2010;137(4):462-76.

12. Levander $E$, Malmgren $O$. Evaluation of the risk of root resorption during orthodontic treatment: a study of upper incisors. Eur J Orthod. 1988;10(1):30-8.

13. Balducci L, Ramachandran A, Hao J, Narayanan K, Evans C, George A. Biological markers for evaluation of root resorption. Arch Oral Biol. 2007;52(3):203-8.

14. Lamster IB, Ahlo JK. Analysis of gingival crevicular fluid as applied to the diagnosis of oral and systemic diseases. Ann N Y Acad Sci. 2007;1098:216-29.

15. Mah J, Prasad N. Dentine phosphoproteins in gingival crevicular fluid during root resorption. Eur J Orthod. 2004;26(1):25-30.

16. Hart PS, Hart TC. Disorders of human dentin. Cells Tissues Organs. 2007;186(1):70-7.
17. Wassall RR, Preshaw PM. Clinical and technical considerations in the analysis of gingival crevicular fluid. Periodontol 2000. 2016;70(1):65-79.

18. Sönmez G, Koç C, Kamburoğlu K. Accuracy of linear and volumetric measurements of artificial ERR cavities by using CBCT images obtained at 4 different voxel sizes and measured by using 4 different software: an ex vivo research. Dentomaxillofac Radiol. 2018;47(8):20170325.

19. Petrie A, Sabin C. Medical statistics at a glance. 3rd ed. West Sussex: Wiley; 2009.

20. Tarallo F, Chimenti C, Paiella G, Cordaro M, Tepedino M. Biomarkers in the gingival crevicular fluid used to detect root resorption in patients undergoing orthodontic treatment: a systematic review. Orthod Craniofac Res. 2019;22(4):236-47.

21. Owman-Moll P, Kurol J, Lundgren D. Repair of orthodontically induced root resorption in adolescents. Angle Orthod. 1995;65(6):403-8.

22. Levander $\mathrm{E}$, Malmgren $\mathrm{O}$, Eliasson S. Evaluation of root resorption in relation to two orthodontic treatment regimes. A clinical experimental study. Eur J Orthod. 1994;16(3):223-8.

23. Aras B, Cheng LL, Turk T, Elekdag-Turk S, Jones AS, Darendeliler MA. Physical properties of root cementum: part 23. Effects of 2 or 3 weekly reactivated continuous or intermittent orthodontic forces on root resorption and tooth movement: a microcomputed tomography study. Am J Orthod Dentofacial Orthop. 2012;141(2):e29-37.

24. Neves FS, de Freitas DQ, Campos PS, de Almeida SM, Haiter-Neto F. In vitro comparison of cone beam computed tomography with different voxel sizes for detection of simulated external root resorption. J Oral Sci. 2012;54(3):219-25.

25. SousaMelo SL, de Vasconcelos KF, Holton N, Allareddy V, Allareddy V, Tabchoury CPM, et al. Impact of cone-beam computed tomography scan mode on the diagnostic yield of chemically simulated external root resorption. Am J Orthod Dentofac Orthop. 2017;151(6):1073-82.

26. Brezniak N, Wasserstein A. Orthodontic root resorption: a new perspective. Angle Orthod. 2016;86(6):1056-7.

\section{Publisher's Note}

Springer Nature remains neutral with regard to jurisdictional claims in published maps and institutional affiliations.

\section{Submit your manuscript to a SpringerOpen ${ }^{\circ}$ journal and benefit from:}

- Convenient online submission

- Rigorous peer review

- Open access: articles freely available online

- High visibility within the field

- Retaining the copyright to your article

Submit your next manuscript at $\boldsymbol{\nabla}$ springeropen.com 